measures that were implemented included installing chlorine injection devices, removing areas of low flow (deadlegs) in the potable water plumbing, disconnecting the water softeners, and repeating the hyperchlorination procedures. No new cases have been identified since September 1996.

In a separate investigation in hospital B in Ohio, nosocomial LD occurred in two patients during January to June 1996. Since 1989, as part of surveillance for nosocomial LD in this hospital, urine samples from all patients with nosocomial pneumonia are tested for Lp-1 antigen. Following the identification of these two cases, an investigation identified a total of 38 cases ( 9 definite and 29 possible) of nosocomial LD. Although information about exposure to showers, aerosol sources, or ingested water was incomplete, in a case control study, cases were more likely than controls to have documented exposure to common aerosol-producing devices (showers or medication nebulizers) during the 2 weeks before onset. Medication nebulizers in this hospital sometimes were rinsed with tap water between doses to reduce clogging.

Lp-1 was isolated from samples obtained from multiple sites in the hot-water distribution system during 1994 to 1996. All Lp-1 isolated from potable hot-water samples in testing done from 1994 to 1996 and in 1984 were identical to the three clinical isolates from 1992, 1994, and 1995. Periodic cultures of the hot-water distribution system were used to guide decontamination efforts.

Thermal heating (to $160^{\circ} \mathrm{F}$ at the tap for 5 minutes) and chlorine decontamination (maintaining a chlorine level of 1 to $2 \mathrm{mg} / \mathrm{L}$ at the tap for at least 5 minutes) had been only temporarily effective in reducing the number of positive sites. A copper-silver ionization system installed in 1985 neither reduced the number of positive samples nor terminated transmission. Additional interventions were initiated, including discontinuing the use of tap water to rinse medical nebulizer equipment, repeating the hyperchlorination procedures as needed in response to positive potable water cultures, increasing the hot-water temperature at the point of use to at least $120^{\circ} \mathrm{F}$, and identifying deadlegs in the potable water plumbing. Following these interventions, no new cases were identified until February 1997; Lp-1 isolates from this patient were identical to all previous isolates. A previously undocumented cross-connection between the hot-water tank from an adjacent outpatient building and the critical-care unit was discovered. After this tank was cleaned and the supply system hyperchlorinated, no new cases have been identified since March 1997.

These reports indicate the capacity for legionellae to colonize hospital plumbing systems for long periods and represent an ongoing risk for infection. Colonization rates are higher in large hospitals with older, large hot-water tanks in which water is held at lower temperatures. Growth of legionellae to high concentrations occurs most often at water temperatures of $77^{\circ} \mathrm{F}$ to $108^{\circ} \mathrm{F}$. Concern about the risk for scalding injuries has prompted some jurisdictions to regulate temperatures in hospital hot-water systems at levels conducive to legionellae growth. Additions and alterations to hospital plumbing systems in response to chang- ing hospital facility needs may create areas of stagnation and sediment build-up, factors also shown to enhance legionellae colonization. These stagnant areas may be resistant to chlorination, thermal disinfection, and ionization. Because eradication of legionellae from water distribution systems generally is not possible, a maintenance program to minimize regrowth of legionellae also should be implemented. Raising the water temperature to $120^{\circ} \mathrm{F}$ to $125^{\circ} \mathrm{F}$ at the fixture (higher temperatures may increase risk of scalding) and infusion of chlorine to maintain consistent levels of 1 to $2 \mathrm{mg} / \mathrm{L}$ have been employed to achieve long-term decontamination in many hospitals. Although metal ionization systems may be effective, it is not clear whether they offer any advantage over conventional methods.

Respiratory therapy equipment should be rinsed only with sterile (not distilled) or tap water.

FROM: Centers for Disease Control and Prevention. Sustained transmission of nosocomial legionnaires' disease-Arizona and Ohio. MMWR 1997;46(19):416-421.

\section{An Outbreak of Enterobacter hormaechei}

Wenger and co-investigators from CDC's Hospital Infections Program and the Hospital of the University of Pennsylvania recently reported an outbreak of Enterobacter hormaechei infections among premature infants in the intensive-care nursery (ICN) of a tertiarycare hospital.

Ten premature infants were found to be culture positive for $E$ hormaechei (six were infected and four colonized) between November 29, 1992, and March 17, 1993.

All isolates were resistant to ampicillin and gentamicin. Isolates from the six infected infants were susceptible to aztreonam, ceftazidime, ceftriaxone, ciprofloxacin, imipenem, and trimethoprim-sulfamethoxazole. All isolates were resistant to cephalothin, piperacillin, and tobramycin.

A cohort study showed the 10 case patients had a lower median estimated gestational age and birth weight than did other ICN infants. Environmental cultures of isolettes and a doorknob in the ICN were positive for the organism. The environmental and case-patient $E$ hormaechei isolates had identical pulsed-field gel electrophoresis band patterns. During the investigation, a number of breaks in infection control practices were observed. These included failure to use gloves, failure to change gloves or gowns between patients, and failure to wash hands or use proper handwashing techniques. Although handwashing facilities were adequate, soap and towels were not always available.

Control measures included the use of contact isolation for culture-positive infants in a common cohort room, additional inservice education of staff, and increased attention to cleaning environmental surfaces (eg, isolette tops).

The outbreak ended after patients were isolated from other infants, adherence to infection control was increased, cleaning of environmental surfaces was enhanced, and empirical antimicrobial covered was changed. 
The authors concluded that environmental contamination and lapses in infection control techniques may have facilitated transmission from patient to patient by healthcare workers. In addition, resistance to ampicillin and gentamicin, the antimicrobials used to treat infants with suspected sepsis in this ICN empirically, may have enabled $E$ hormaechei to become epidemic.

$E$ hormaechei first was identified as a unique species in 1989; however, this is the first published report of clinical infections due to this organism.

FROM: Wenger P, Tokars J, Brennan P, et. al. An outbreak of Enterobacter hormaechei infection and colonization in an intensive care nursery. Clin Infect Dis 1997;24: 1243-1420.

\section{Antimicrobial Control Programs Result in Annual Savings Up to $\$ \mathbf{5 0 0 , 0 0 0}$}

The current cost of antimicrobial therapy is more than $\$ 7$ billion annually in the United States, with up to $\$ 4$ billion used for treatment of nosocomial infections due to antibiotic-resistant bacteria. Antimicrobials account for up to $30 \%$ of hospital drug budgets. Moreover, it has been recognized for more than 3 decades that up to $50 \%$ of antimicrobial usage in US hospitals is inappropriate. Now that managed-care organizations oversee a greater proportion of healthcare expenditures, there is even greater fiscal pressure to limit antimicrobial costs.

In this era of return to primary care, infectious disease (ID) physicians need to provide evidence that their nonpatient-care activities remain cost-effective. As such, it is important to document the crucial role of the infection control practitioner (ICP) in programs to control antimicrobial costs.

Researchers from the Robert Wood Johnson Medical School and the University of Pennsylvania School of Medicine recently reviewed a representative sample of articles from 1966 to 1995 that dealt with antimicrobial costs. The methodology and cost effectiveness of antimicrobial control programs in the United States and Canada were reviewed to determine the role of the ICP in designing and implementing these programs to make recommendations that maximize the impact that ID physicians could have on containing hospital antimicrobial costs.

Controlling antimicrobial costs was found to be achieved by the use of eight strategies: education, formulary restriction, pharmacy justification, formulary substitution, computer surveillance, laboratory item cost listing, purchase plans, and multidisciplinary approaches. Most strategies had input from ID physicians and resulted in cost savings up to $\$ 500,000$ annually, particularly during the initiation periods. Educational efforts were successful in reducing costs but needed continual intervention. Formulary restriction was found to be the most straightforward cost-control mechanism. Restriction of target antimicrobials has given way to switch therapy between expensive and less costly agents or between parenteral and oral regimens. Switch therapy is facilitated through the use of innovative order forms and on-line computer interaction. Computer surveillance has the capacity for interactive controls. Purchase plans may give way to centralized pharmacy monitoring, a strategy that is attractive to managed-care organizations.

In the large representative sample of articles, it was found that an ICP was involved approximately $85 \%$ of the time in either planning or executing an antimicrobial control program. Comprehensive programs, those that used four or more strategies to control costs, usually featured multidisciplinary teams, working together to implement multiple strategies and to obtain the best economic success. The role of hospital administration was highlighted as a key player by recognizing the benefits of new strategies and the need for designation of leaders to implement the plans.

The authors point out that the function of controlling antimicrobial costs can be usurped quickly by other healthcare providers, including pharmacists and quality-care administrators. Step-by-step details are provided for the ID physician to translate his or her expertise in antimicrobial use into leadership of the antimicrobial management program. For example, the authors suggest that it is important to understand the goals of the managed-care organizations (MCOs) whose focus is on capitated care and savings on all pharmaceuticals, particularly antimicrobials for both inpatients and outpatients. The MCOs may wish to develop their own teams for antimicrobial management programs that serve several hospitals and may be in a better position than smaller hospitals to provide the initial capital outlay for developing such programs and may have a large centralized data processing center to analyze data. Formularies already published by large pharmaceutical claim companies, some insuring up to 59 million lives, should be attractive to MCOs. The ID physicians should approach these consortiums to develop incentive programs based on expenditures for both outpatient and inpatient drugs and devices used to treat infections.

The authors conclude that the ID physicians are best equipped to address cost-containment issues and need to mobilize their colleagues in pharmacy, nursing, and quality management to present a comprehensive and cohesive program to hospital administration that will appeal to the MCOs in their region. They direct the ID physician to use the numerous published algorithms (termed critical pathways and clinical practice guidelines) as a checklist or road map for contemporary implementation of antimicrobial control programs.

FROM: John JF, Fishman NO. Programmatic role of the infectious disease physicians in controlling antimicrobial costs in the hospital. Clin Infect Dis 1997;24:471-485.

Additional news items in this issue: NIOSH Publishes Latex Allergy Alert, page 537; Hepatitis B Outbreak in EEG Clinics in Toronto, page 553; Nosocomial Aspergillosis Associated With Construction, page 560; Free Access to MEDLINE on $W W W$, page 565; TB Skin Testing of BCG-Vaccinated Children, page 574. 